NASA Technical Memorandum 103607

\title{
A Failure Diagnosis System Based on a Neural Network Classifier for the Space Shuttle Main Engine
}

Ahmet Duyar

Florida Atlantic University

Boca Raton, Florida

and

Walter Merrill

National Aeronautics and Space Administration

Lewis Research Center

Cleveland, Ohio

Prepared for the

29th Conference on Decision and Control

sponsored by the Institute of Electrical and Electronics Engineers

Honolulu, Hawaii, December 5-7, 1990 


\section{A FAILURE DIAGNOSIS SYSTEM \\ BASED ON A NEURAL NETWORK CLASSIFIER \\ FOR \\ THE SPACE SHUTTLE MAIN ENGINE}

\author{
Ahmet Duyar \\ Mechanical Engineering Department \\ Florida Atlantic University \\ Boca Raton, Florida 33431
}

\author{
Walter Merrill \\ NASA Lewis Research Center \\ Cleveland, Ohio 44135
}

\begin{abstract}
A conceptual design of a model based failure detection and diagnosis system is developed for the space shuttle main engine. This design relies on the accurate and reliable identification of the parameters of the highly nonlinear and very complex engine. The design approach was presented in some detail and results for a failed valve are presented. These preliminary results verify that the developed parameter identification technique together with a neural network classifier can be used for this purpose.

\section{INTRODUCTION}

This paper describes a model based failure diagnosis system based on a neural network classifier for the space shuttle main engine (SSME).
\end{abstract} The system may be used to monitor the life cycle of engine components and for the early detection, isolation and the diagnosis of engine failures. As such, the proposed system will be one part of a larger, engine health monitoring system [1]. The health monitoring system will allow for accommodation of failures, reduce maintenance cost, increase engine availability, and be one part of an integrated, intelligent control system [2] for the SSME. A description of SSME dynamics and its modeling is given in a study by Duyar, Guo and Merrill [3]. A summary of the major failures of the SSME that have occurred are outlined by Cikanek [4]. Several authors survey $[5,6,7,8]$ the available methods and approaches for failure detection and diagnosis. In particular the survey by Isermann[6] gives several examples of the use of identification techniques for process failure detection.

A failure is the abnormal behavior of a component due to physical change in the component. A failure event often impairs or deteriorates the system's ability to perform its specified tasks or mission. The derection task is defined as the act of identifying the presence of an unspecified failure. After a failure is detected, then the failure must be isolated to the component that has failed. During the process of isolation the magnitude of the failure may be estimated. Failure diagnosis is the isolation and estimation of a failure mode. Once a failure is detected and diagnosed, the failure can be accommodated through reconfiguration of the system. Reconfiguration includes both hardware actions (e.g., activating back - up systems) and software tasks (e.g., adjusting the feedback control gains). The detection and diagnostic tasks may be accomplished by an on board processor, on line and in real time for failure accommodation, as well as by an off line processor which analyzes recorded data for life cycle analysis and preventive maintenance.

Initially a brief description of the conceptual design of the model based failure detection and diagnostic system (FDDS) is given. This is followed by a description of the method used to design the detection and diagnostic system. The design is applied to the detection of simulated data of a stuck valve to demonstrate the performance of the FDDS.

\section{CONCEPTUAL DESIGN}

Model based failure detection methods rely on the determination of changes appearing in the system due to the existence of a failure, in comparison to the normal status of the system. For example, in aerospace applications the failure of control actuators may manifest themselves as shifts in the parameters of control gain matrix. Failures of sensors may take the form of abrupt changes in the parameters of the output matrix, or increases in measurement noise. These changes are determined by comparing the parameters of the observed process with the parameters obtained from the model of the normal process. The differences between these parameters are called residuals. The 
residuals and their patterns are analyzed for failure detection and diagnosis by comparing them with the known failure signatures of the process.

Failure signatures, which show the effect of a failure on the parameters, are generated by inducing failures in the performance model of the process. Failure diagnosis is accomplished by training a neural network classifier to recognize the pattern of the respective failure signatures.

The design of the FDDS is accomplished in three stages: process modeling, residual generation and failure detection and diagnostic classifier design. In the following sections the methods used in these stages are briefly explained.

\section{PROCESS MODELLING}

A complete nonlinear dynamic simulation of SSME performance was developed by Rocketdyne Division of Rockwell International Corporation[9]. In this study, this nonlinear model is considered as the unknown process. It is used for the generation of failure signatures by modifying the actuator models to simulate failure. The input output data generated from this simulation is also used to identify the parameters of the engine. Due to its size and complexity ( $40 \mathrm{~min}$. CPU time for $20 \mathrm{sec}$. of real time operation with a VAX 8800), this nonlinear simulation cannot be used to generate data in real time to describe the normal mode of operation.

An off-line system identification algorithm developed by Eldem and Duyar [10] and the data generated from the nonlinear performance simulation are used to obtain linear point models of the SSME at twenty five different operating points. The inputs of these models are the rotary motion of the valve actuator outputs of the oxidizer preburner oxidizer valve (OPOV), $\beta_{\text {OPOV }}$, and fuel preburner oxidizer valve (FPOV), $\beta_{\text {Fpov }}$. The point models have measurable state variables which make them more suitable for failure detection and diagnostic studies, since the need for state estimation is eliminated. The outputs which are also the state variables, are the chamber inlet pressure, $P_{C}$, mixture ratio, MR, high pressure fuel turbine speed, $S_{\mathrm{HPFT}}$, and high pressure oxidizer turbine speed, $S_{\text {HPOT. }}$.

Two models of the SSME are utilized in the design of the FDDS: a linear state variable observer or state variable filter, and a linear state space model of the normal operation of the engine.

Consider the discrete state space representation of the engine, linearized about one operating point.

$$
\begin{aligned}
& x(n+1)=A x(n)+B u(n) \\
& y(n)=C x(n)
\end{aligned}
$$

where $x, u$ and $y$ are the deviations of the state, the input and the output vectors about an operating point. For mathematical simplicity, it is assumed that the system is not subject to disturbances and to sensor noise. Following the work of Eldem and Duyar [10], it is assumed that the system is in $\alpha$ canonical form.

To estimate the states from the measured input and output data, a state variable filter

$$
\begin{aligned}
& x_{f}(n+1)=A x_{f}(n)+B u(n)+K\left[y(n)-C x_{f}(n)\right] \\
& y_{f}(n)=C x_{f}(n)
\end{aligned}
$$

is used. Here the subscript $f$ denotes the estimated values obtained from the filter. The observer gain matrix $\mathrm{K}$ is selected as a deadbeat observer gain satisfying the following relations:

$$
\begin{aligned}
& C=\left[0: H^{-1}\right] \\
& A=A_{0}+K H C \\
& A_{0}{ }^{\mu}=0 \\
& (H C)_{i} A_{0}{ }^{\mu j}=0 \\
& (H C)_{1} A_{01} K^{j}=0,0 \leq 1<\left(\mu_{1}-\mu_{j}\right)
\end{aligned}
$$

Here the subscript $i$ and $j$ denote the $i$ 'th row and $j$ 'th column respectively. Here $A_{0}$ is a lower left triangular structure matrix which consists of zeros and ones only and is determined by the observability indices, $\mu_{k}$ where $k$ associates $\mu_{k}$ with the $k$ 'th output. Using these relations, Eqs. 1 and 2 can be solved to give

$$
x_{f}(n)=A_{0}{ }^{n} x(0)+\Sigma A_{0}^{i-1}[K H: B] \underset{|u(n-i)|}{|y(n-i)|}
$$

where, $\mu=\max \left(\mu_{1}\right)$. Using the nillpotency of $A_{0}$ the above equation yields the state variable filter equation

$$
\begin{aligned}
& x_{r}(n)=\Sigma A_{0}{ }^{i-1}[K: B] \\
& |y(n-i)| \\
& \text { for } n>\mu(n-i) \mid \\
& y_{r}(n)=C x_{d}(n)
\end{aligned}
$$

which can be used to estimate the states from the measurements of the input and the output data. 
Unlike the state variable filter the state space model of the engine estimates the state variables from the measurement of the input data only. The equation describing the state space model of the engine is given below:

$$
x_{m}(n)=\Sigma A_{0}^{1-1}[K: B]\left|y_{m}(n-i)\right|
$$

for $n>\mu$

$$
y_{m}(n)=C x_{m}(n)
$$

Here the subscript $m$ denotes the variables estimated by the model.

Both the state variable filter and the state space model are tested by comparing their prediction with the actual output obtained from the nonlinear simulation. The point models can predict the output of the nonlinear simulation with very good accuracy [11]. These point models are linked to obtain a simplified quasi linear model of the SSME, valid within its full range of operation [12]. The parameters of the point models are regressed with the parameters determining the nominal operating conditions. The mixture ratio and the chamber pressure are considered as the parameters which determine a nominal operating condition. This simplified model is also tested by designing an input signal as shown in Figure 1. The comparison of the responses of the linked model and the nonlinear simulation again indicated good agreement as shown in Figures 2 and 3. Table 1 presents the standard error of estimates.

\section{RESIDUAL GENERATION}

As mentioned earlier, it is assumed that failures are indicated by changes in the parameters of the system as well as by internal, observable, but not necessarily measured, process state variables. The state variables can be estimated by a filter or a state space model based on the known process parameters. The parameters of the process can be determined by using a system identification technique. Then, residuals can be generated by taking the difference between the actual parameters and the observed parameters.

Two kinds of residuals may be generated: 1)parameter, by comparing the identified parameters of the engine with the normal parameters, and 2)output, by taking the difference between the actual output and the output obtained from the estimated state variables. The simplest test to detect a failure is a comparison of residual magnitudes to a threshold value. Using the distribution of the variances of the residuals under failure free conditions, the threshold values can be determined to minimize false alarms and missed detections using the Neyman-Pearson criterion 13.

In this study, the state variables of the system are used for the generation of output residuals. With the observed state variables, residuals are generated between the measured output and the output obtained using the observed state variables as:

$$
\delta y_{1}(n)=y(n)-C x_{r}(n)
$$

Measured output and the output obtained from the state space model are also used to generate additional residuals as:

$$
\delta y_{2}(n)=y(n)-C_{m} x_{m}(n)
$$

Here subscript e denotes the estimated values.

These residuals are generated by inducing stuck valve failures in the nonlinear dynamic simulation of the SSME. Both the OPOV and the FPOV are considered for this purpose. Data are obtained at various angles for which these valves are stuck. Figure 4 shows the residuals obtained for two of the outputs, chamber inlet pressure and the mixture ratio, by using Equation 12.

\section{FAILURE DETECTION AND DIAGNOSIS}

A neural network classifier is used for failure detection and diagnosis purposes. Following the work of Dietz, Kiech and Ali [14], a two layer network architecture combined with a back propagation algorithm is selected. Top level neural networks detect the existence of a failure and classify the type of failures. The lower level networks classify the severity of the failures after their type are determined at the top level.

In the example considered in this paper, output residuals obtained in the previous section are used to train the network to detect and diagnose OPOV and FPOV stuck valve failures. Two top level networks are used to classify the failure type as shown in Figure 5. One uses the chamber pressure residual as its input, the other, mixture ratio residual. Each classifier network has two output nodes, each of which is associated with either an OPOV stuck or FPOV stuck condition. During training, a residual pattern representing a failure condition is applied to the input level and a 1 
indicating complete activation is compared to the corresponding output node. Back propagation is used to adjust the network weightings. Two second level networks, one for each failure, are used to determine the setting, in degrees, at which the valve is stuck. Each lower level network has three output nodes corresponding to different levels of severity, that is valve opening, and were trained in a similar manner. Each of the four networks has 200 input nodes and $\mathbf{2 0}$ hidden layer nodes in their architecture. The input to each network is a time sequence of residuals of length 200 . The time step between residuals is 0.04 secs with the total sequence time representing 8 secs.

Test data with severity levels not used in training were used with the networks with successful results. Figure 6 and 7 illustrate the results obtained for inputs of residual patterns for OPOV stuck at $\mathbf{4 7 . 5}$ degrees and FPOV stuck at 55 degrees. In both cases the networks correctly identify both the failure types and their severity.

\section{CONCLUSION}

A conceptual design of a model based FDDS is developed for the SSME. This design relies heavily on the accurate and reliable identification of the parameters of the highly nonlinear and very complex SSME. The design approach was presented in some detail and preliminary results for a failed valve were presented. These preliminary results verify that the developed parameter identification technique can be used for this purpose. Additional simulation studies are needed, using the failure data generated with the nonlinear simulation, to completely verify the validity of the failure detection concept presented in the paper. Also, additional research is required to incorporate more failure modes.

Both the point models and the state variable filters provide accurate representation of the nonlinear simulation within acceptable error limits. However, research continues to improve their accuracy by identifying nominal models using multi-level, pseudo-random sequences as the driving signals. Further study is also needed to determine the capability of the neural network to recognize failure patterns for failure diagnosis purposes.

\section{REFERENCES}

1. Guo, Ten-Huei and Merrill, Walter C.: A Framework for Real-Time Rocket Engine Diagnostics. Presented at the 1990 NASA Conference on Advanced Earth-to-Orbit Propulsion Technology.

2. Merrill, W. C. and Lorenzo, C. F., "A Reusable Rocket Engine Intelligent Control," NASA TM 100963, ALAA-88-3114, July 1988.

3. Duyar, Ahmet; Guo, Ten-Huei; and Merrill, Walter C.: Identification of Space Shuttle Main Engine Dynamics. IEEE Control Systems Magazine, Vol. 10, No. 4, pp 59-65, June 1990.

4. Cikanek, H. A., "Space Shuttle Main Engine Failure Detection," IEEE Control System Magazine, Vol.6, pp. 13-18, June, 1986.

5. Willsky, A. S., "A Survey of Design Methods for Failure Detection in Dynamic Systems," Automatica, Vol.12, pp.601-611, 1976.

6. Isermann, R., "Process Fault Detection Based on Modelling and Estimation Methods," Automatica, Vol.20, pp.387-404, 1984.

7. Gertler, JJ., "Survey of Model Based Failure Detection and Isolation in Complex Plants," IEEE Control Systems Magazine, pp.3-11, December, 1988.

8. Frank, P.M., "Fault Diagnosis in Dynamic Systems Using Analytical and Knowledge Based Redundancy-A Survey and Some New Results," Automatica, Vol. 26, No.3, pp:459-474, 1990.

9. Rockwell International Corporation, "Engine Balance and Dynamic Model," Report FSCM No. 02602, Spec. No. RLO0001., 1981.

10. Eldem, V. and Duyar, A. "Identification of Discrete Time Mulitvariable Systems; A Parameterization via $\alpha$-Canonical Form," Submitted to Automatica, June 1989. 
11. Duyar, A., Eldem, V., Merrill, W. C. and Guo, T.-H., "State Space Representation of the Open Loop Dynamics of the Space Shuttle Main Engine," Submitted to ASME Journal of Dynamic Systems Measurement and Control, Dec. 1989.

12. Duyar, A., Eldem, V., Merrill, W. C. and Guo, T.-H., "A Simplified Dynamic Model of the Space Shuttle Main Engine," Submitted to ASME Journal of Dynamic Systems Measurement and Control, June 1990.

13. Sage, A. P. and Melsa, J. L.; Estimation Theory with Applications to Communications and Control, McGraw-Hill 1971, pp 116-124.

14. Dietz, W. E., Kiech, E. L. and Ali, M., "Jet and Rocket Engine Fault Diagnosis in Real Time," Journal of Neural Network Computing, pp.5-18,1989.

\begin{tabular}{|c|c|c|c|c|}
\hline \multicolumn{5}{|c|}{ TEST SIGNAL } \\
\hline OUTPUT & $P_{C}$ & MR & $S_{\text {HPFT }}$ & $S_{\text {HPOT }}$ \\
\hline Filter & 0.0068 & 0.0114 & 0.0056 & 0.0037 \\
Model & 0.0151 & 0.0147 & 0.0116 & 0.0095 \\
\hline
\end{tabular}

Table 1 Standard Error of Estimates (SEE) of Predictions of the Filter and the Model For the Test Signal Covering All Operating Points. 


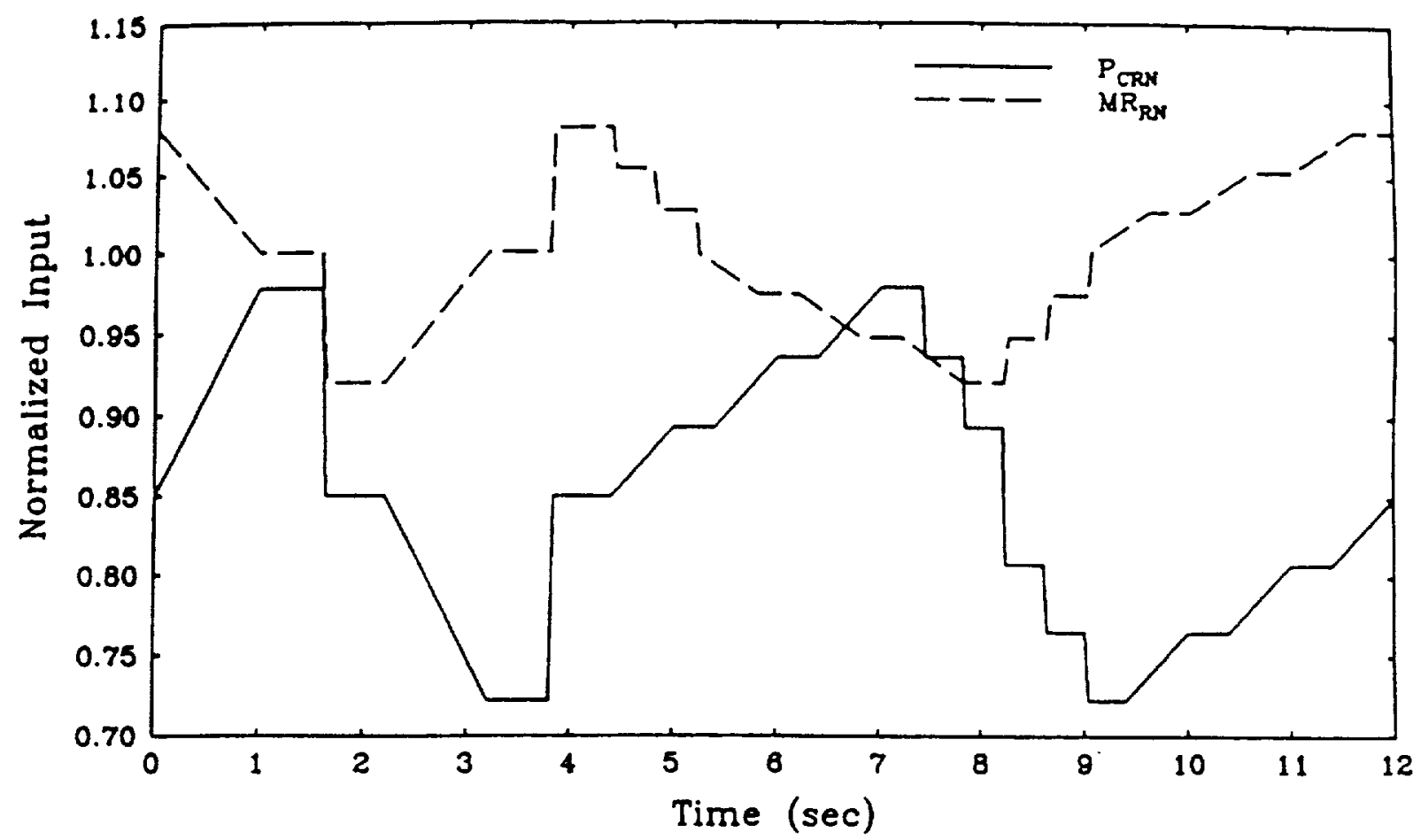

(a) $P_{C R N}$ and $M R_{R N}$ requests.

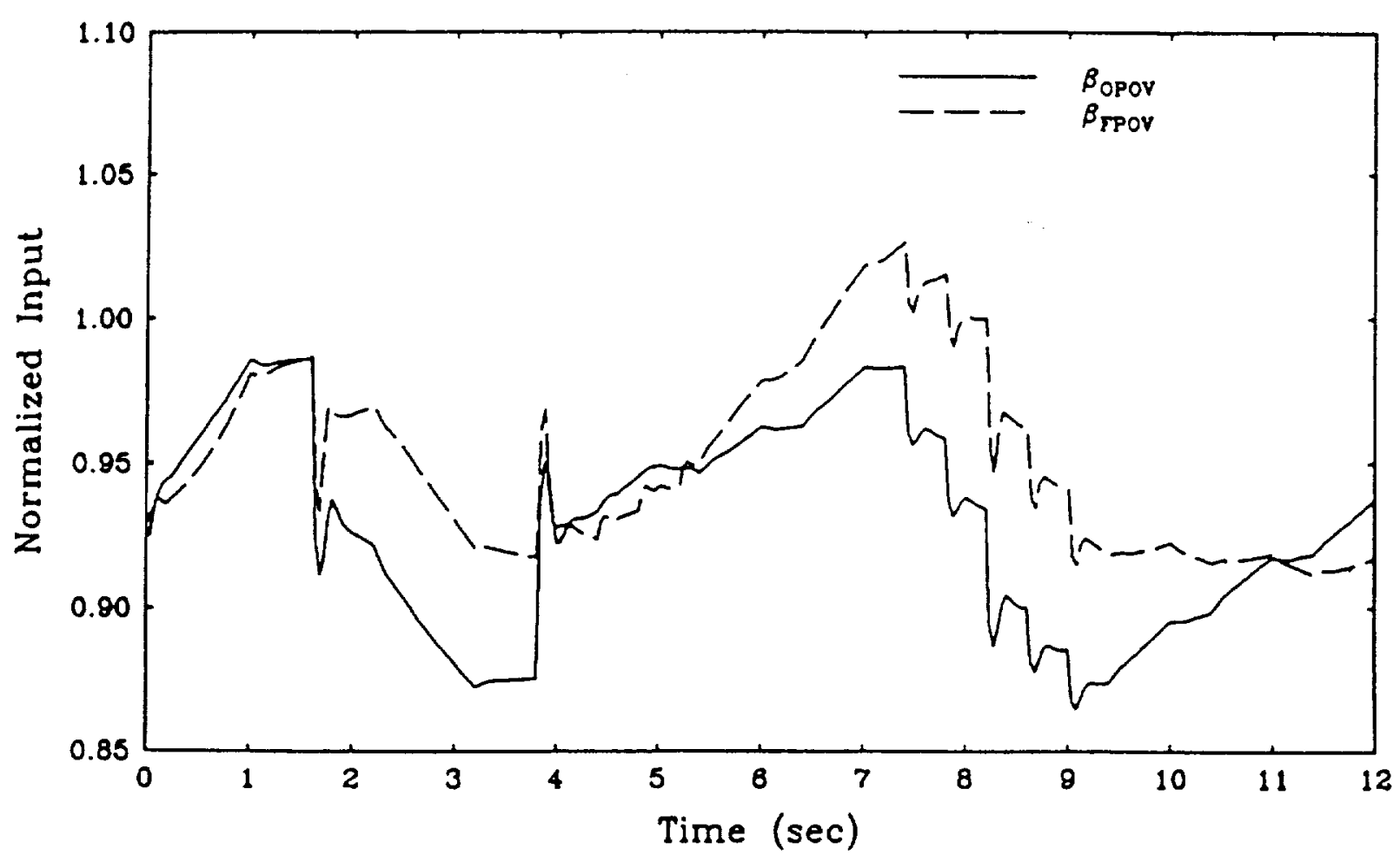

(b) $\beta_{\text {OPOV }}$ and $\beta_{\text {FPOV }}$ signals.

Figure 1.-Reference input signals and their corresponding valve inputs. 


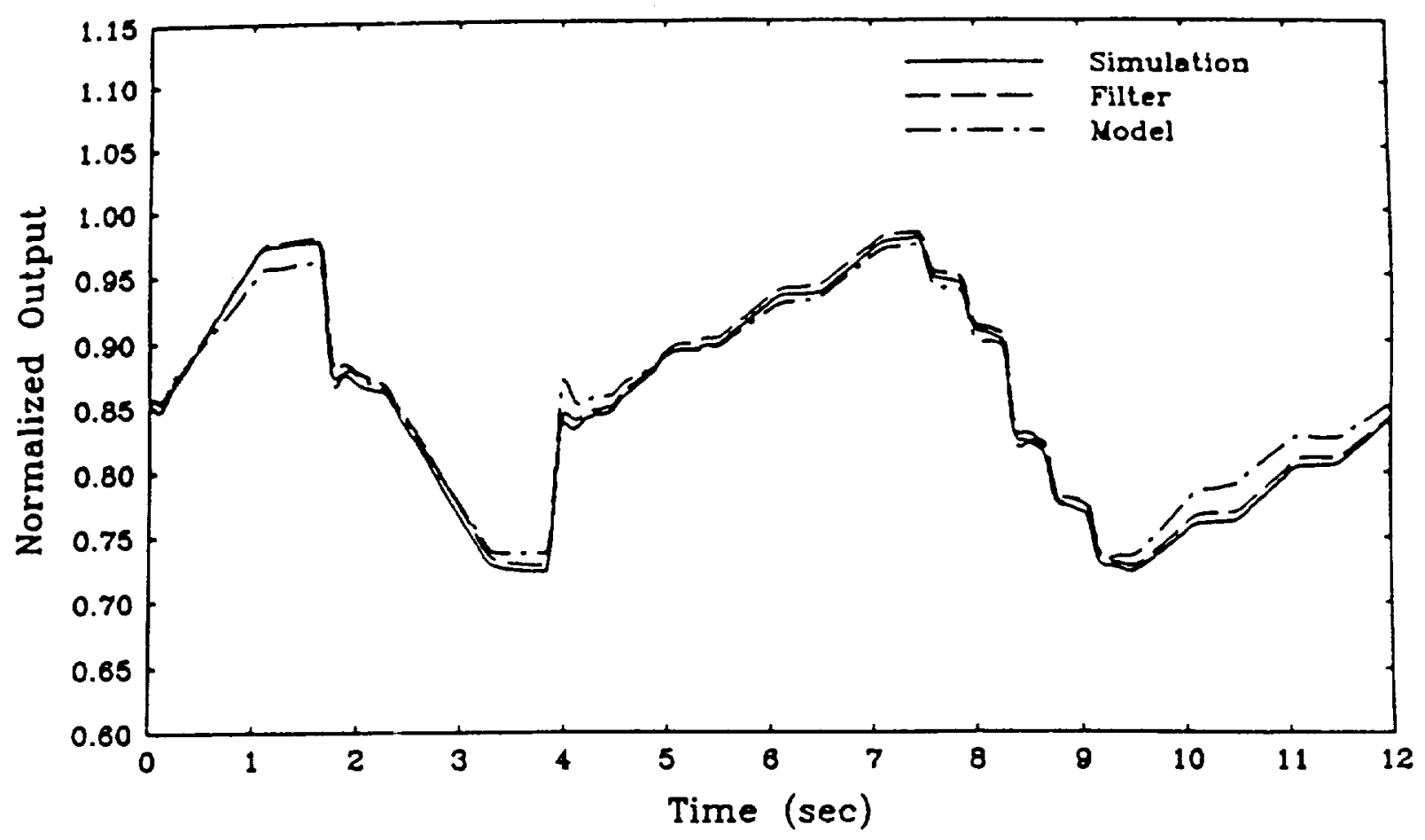

(a) $P_{C N}$ chamber inlet pressure.

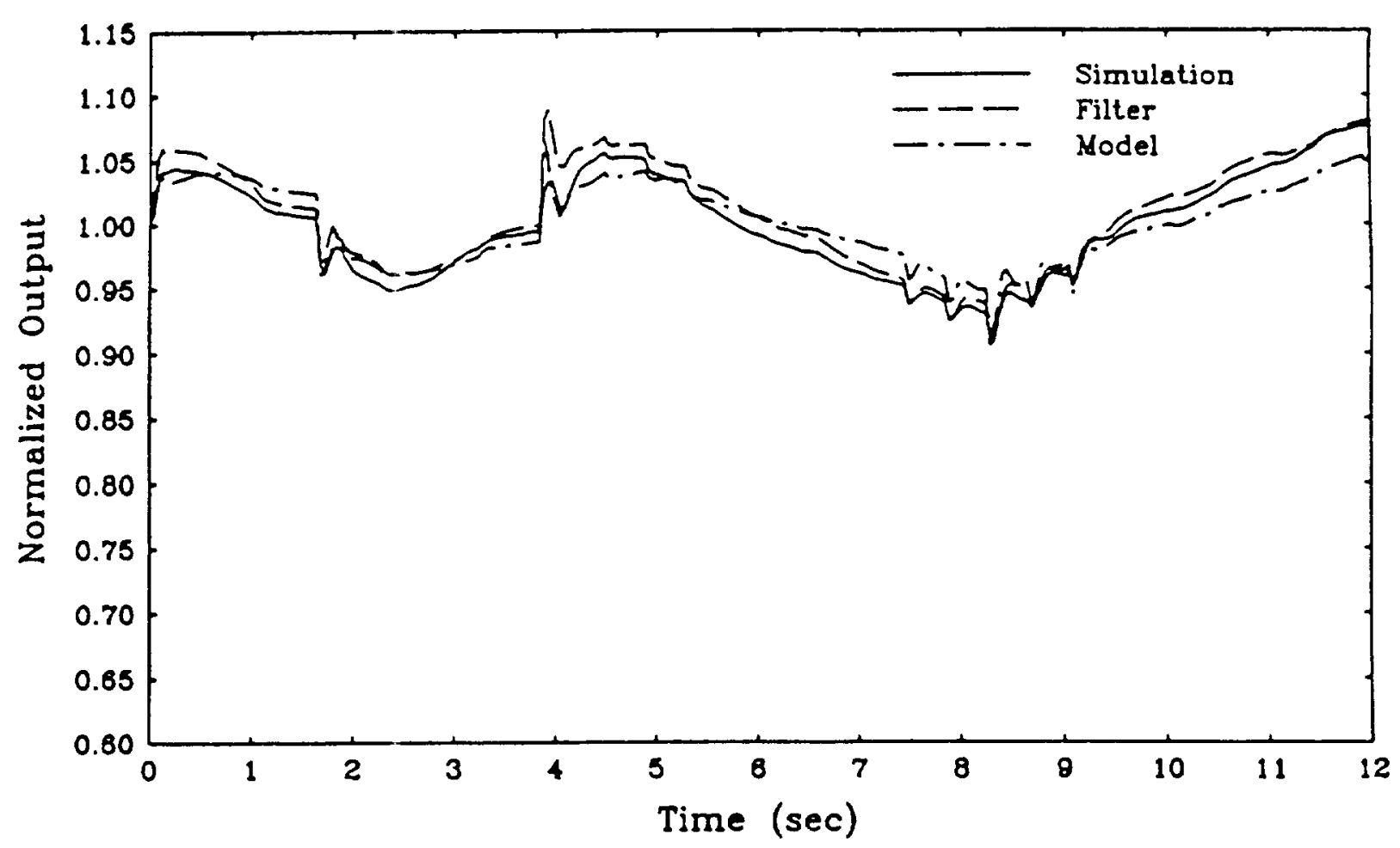

(b) $M R_{N}$ mixture ratio.

Figure 2.--Comparison of the responses of the linked model and the filter with the nonlinear simulation. 


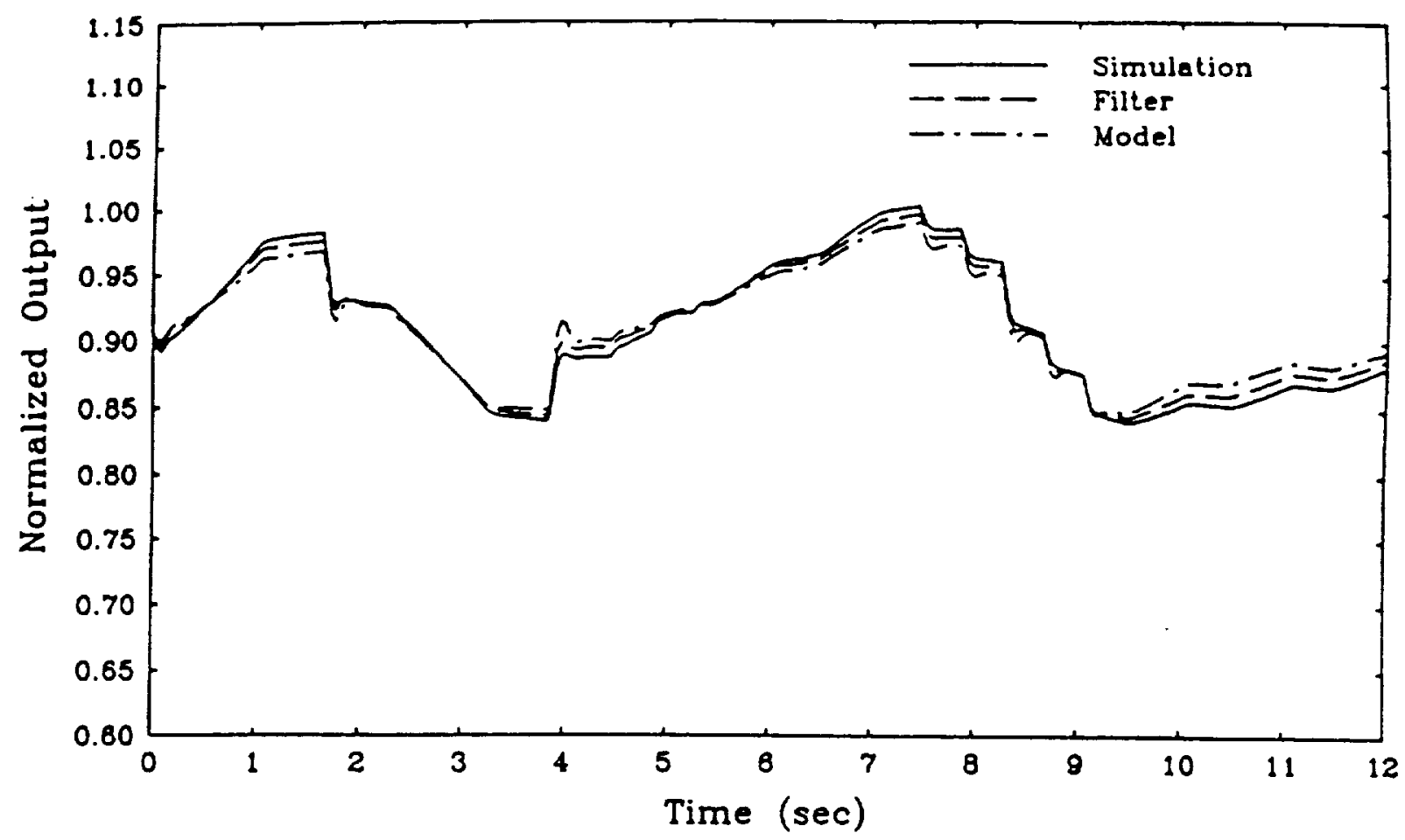

(a) SHPFTN high pressure fuel turbine speed.

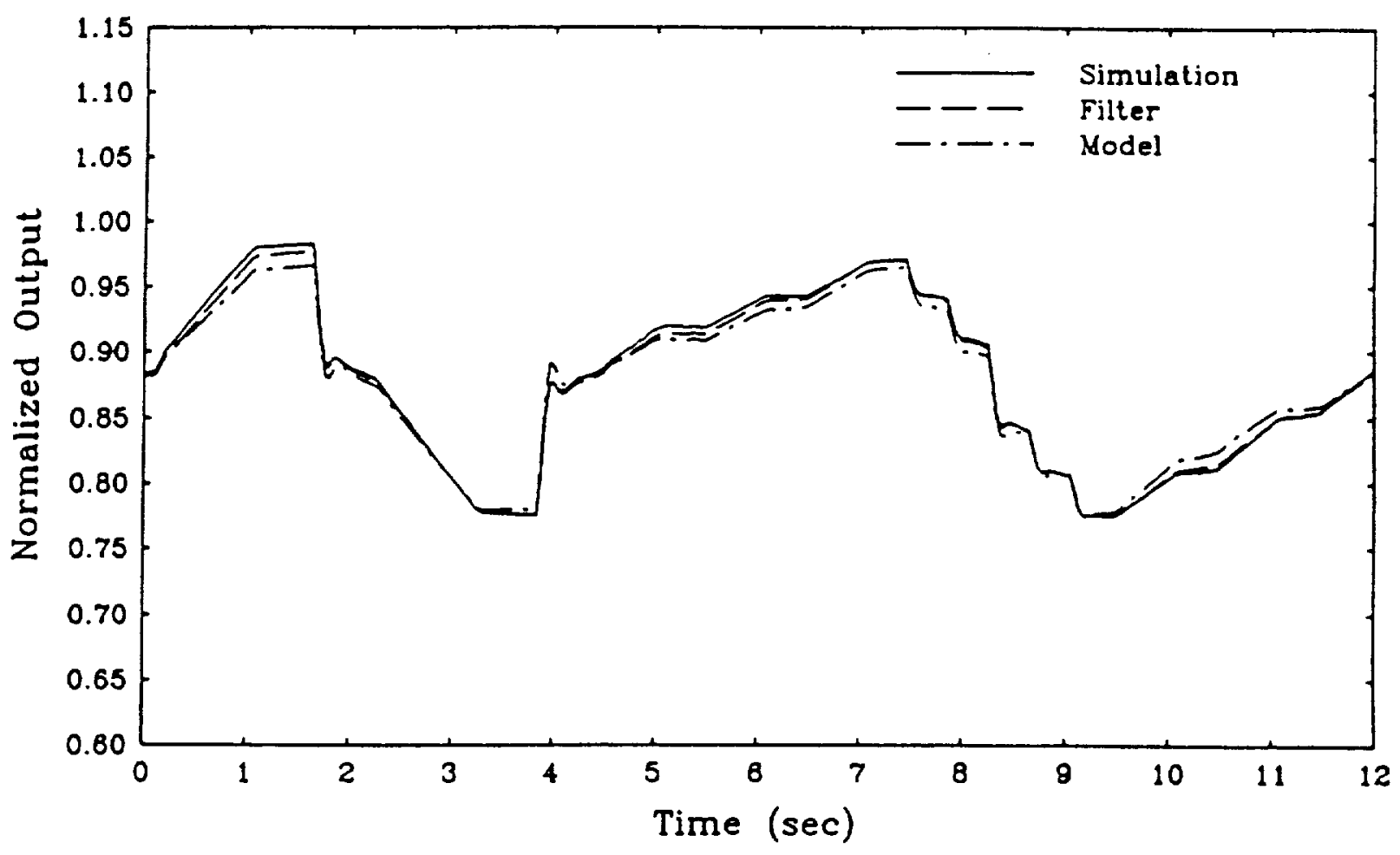

(b) SHPOTN high pressure oxidizer turbine speed.

Figure 3.-Comparison of the responses of the linked model and the filter with the nonlinear simulation. 


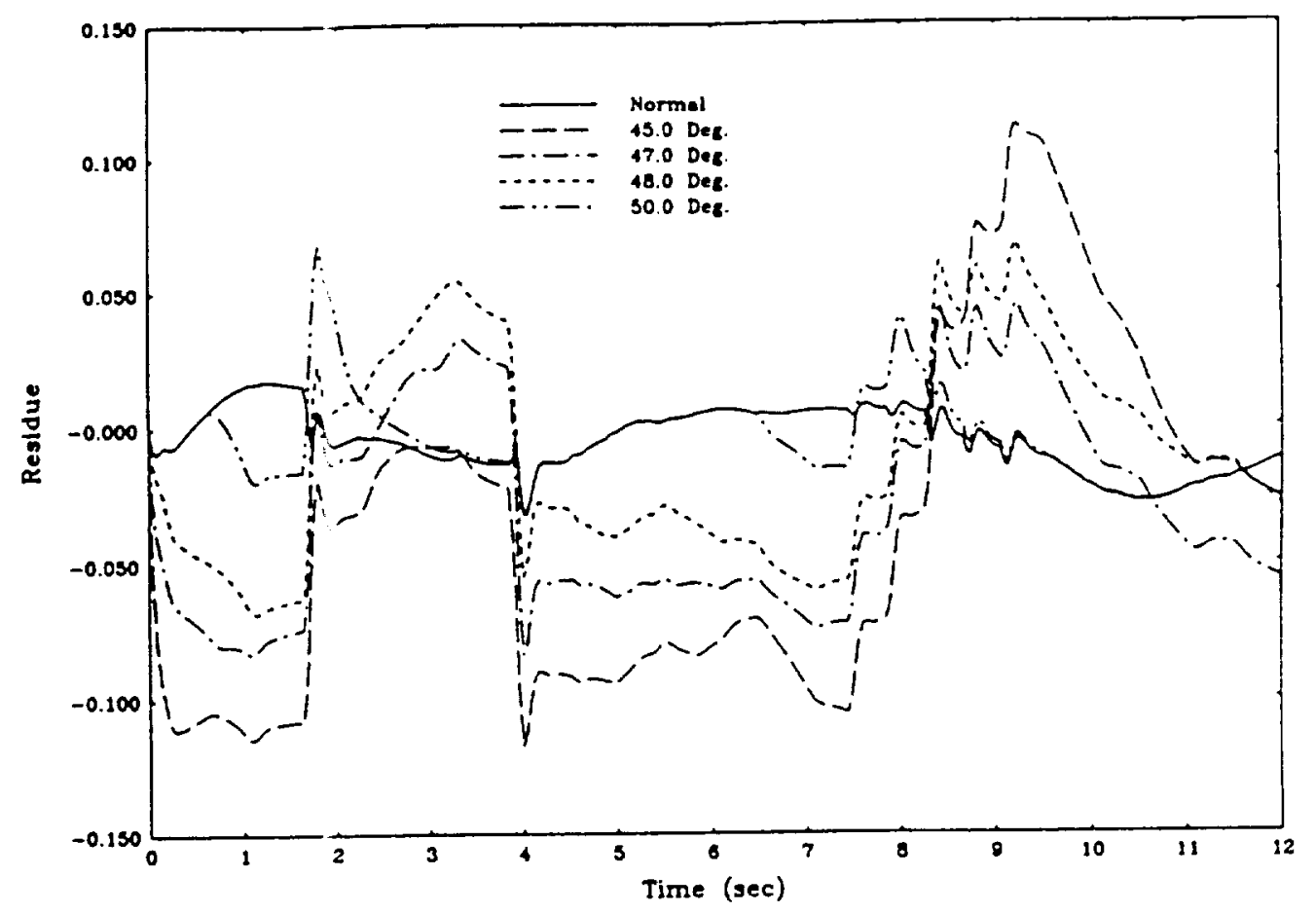

(a) $P_{C}$ residues for OPOV stuck at various angles.

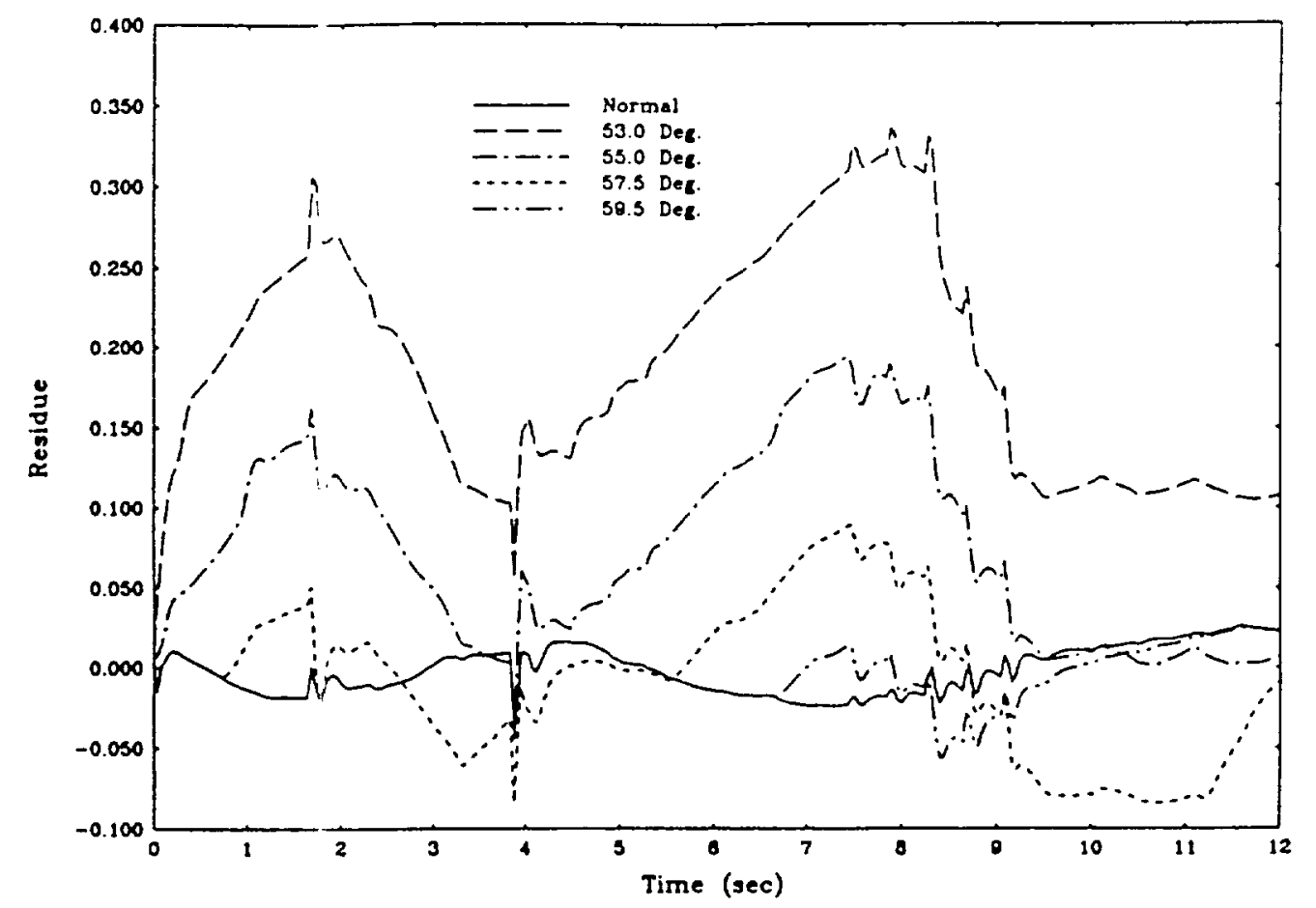

(b) MR residues for FPOV stuck at various angles.

Figure 4.--Output residues for stuck valve failures. 


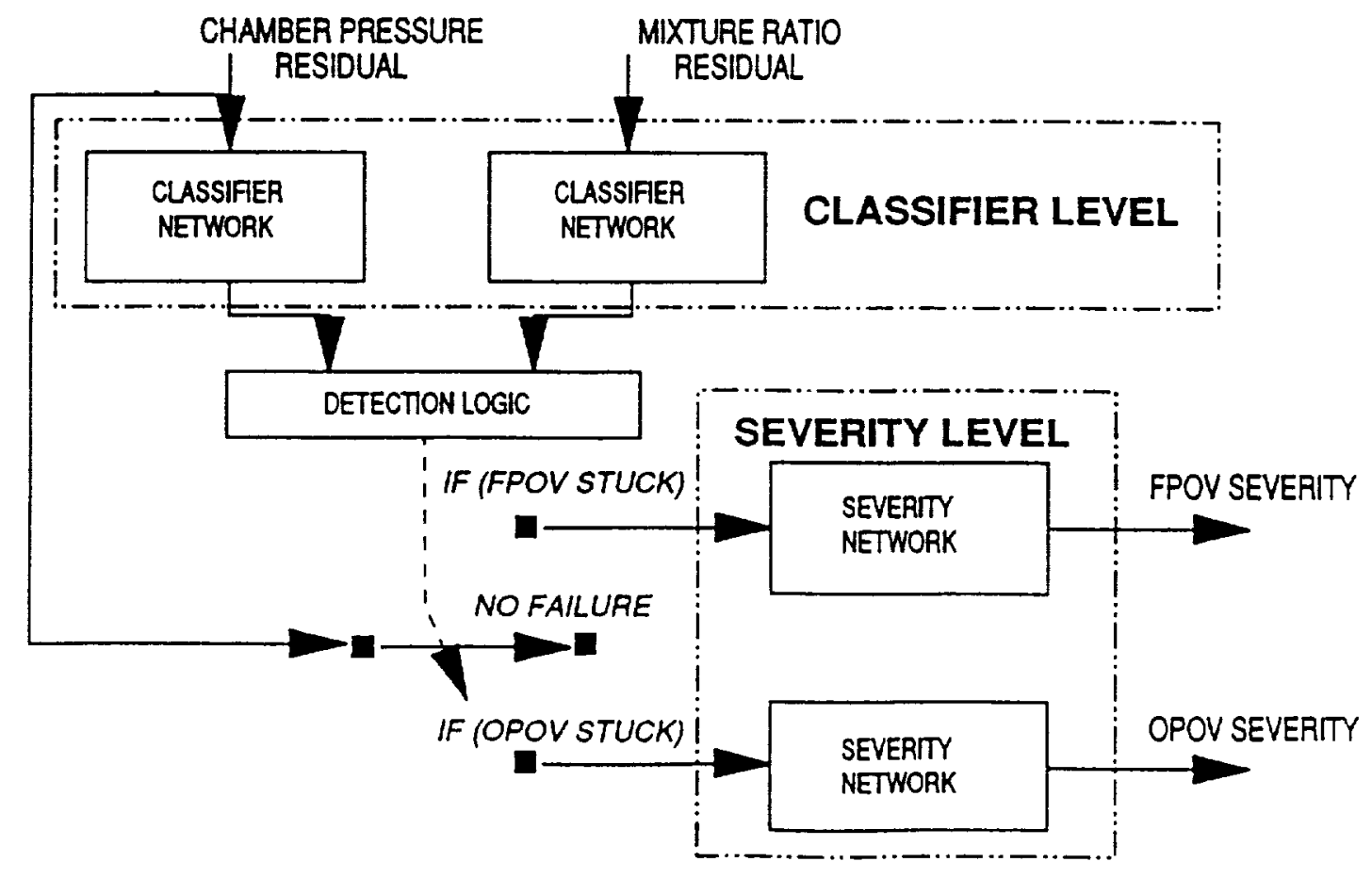

Figure 5.-Neural network architecture diagram.

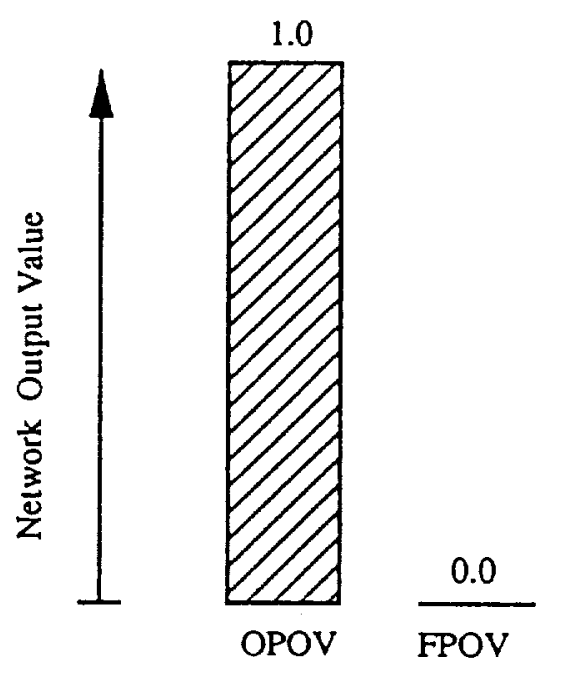

(a) Fault Type
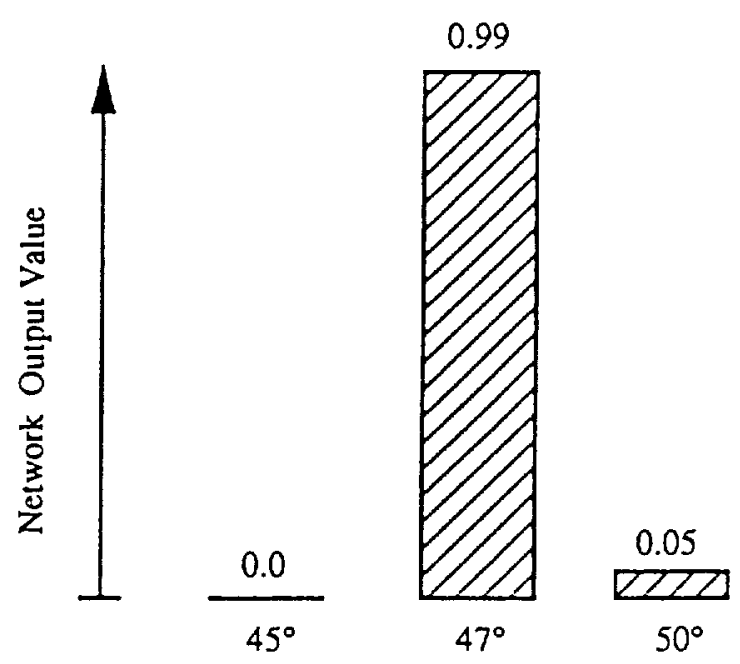

(b) Severity

Figure 6.-Responses of the neural network to OPOV stuck at 47.5 degrees. 


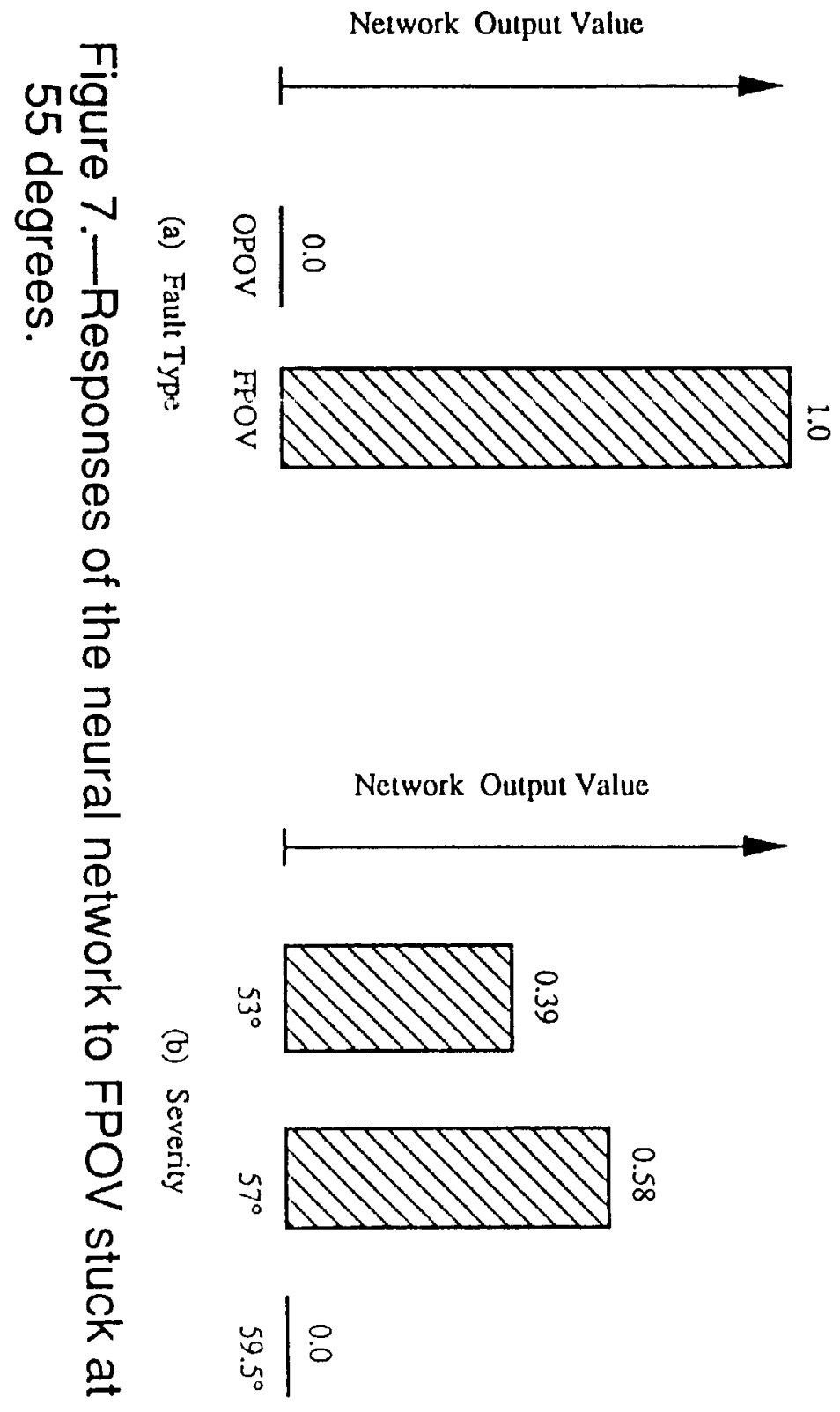




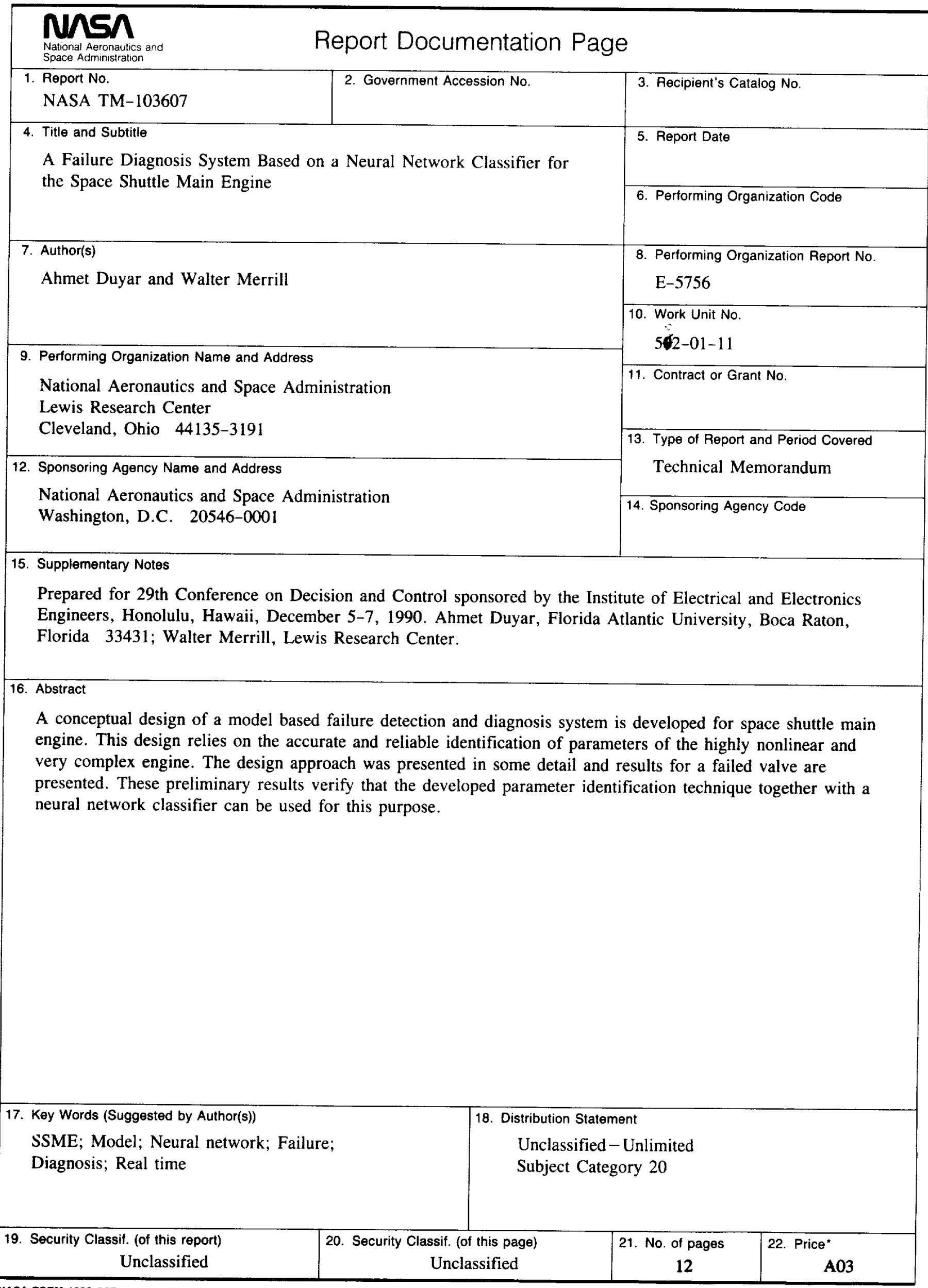

